\title{
PROPAGATION OF SHOCK WAVES IN BUBBLE-LIQUID MIXTURES
}

\author{
L. VAN WIJNGAARDEN \\ Twente Institute of Technology, Enschede
}

\begin{abstract}
In this paper experiments are described on shock waves in a mixture of an aqueous solution of glycerine and small air bubbles. Bubble size, shock strength and volumetric air content are varied during these experiments. Results are given concerning the measured shock velocity, the shock thickness and the occurrence of pressure waves at the back side of the shock. These results are compared with those of previous theoretical work by the author. Good agreement with theory is found regarding shock velocity and shock thickness. The pressure waves predicted by theory do appear indeed, but are attenuated considerably stronger than according to the theory.
\end{abstract}

\section{INTRODUCTYON}

Travelling compressive waves of finite amplitude in a bubble-liquid mixture are subjected to amplitude dispersion, which leads to steepening of the wave, frequency dispersion, having the opposite effect, and dissipation of various kinds. The interaction of these three mechanisms may result in a stationary shock wave, as was shown theoretically in [1]. There the structure of a shock wave in a bubble-liquid mixture was analysed and its propagation speed, thickness and other properties predicted. Only weak and moderately strong shocks were considered in [1]. The theory predicts in particular the occurrence of pressure waves at the back side of the shock.

This contribution has as one objective: to present results of experimental work on shock waves in bubbly suspensions, carried out in our laboratory by L. Noordzij. Another objective is to discuss these results in connection with the theoretical predictions.

In the next section, a brief description of the experimental apparatus is given. In the third section experimental results concerning shock velocity, shock thickness and other aspects (waves at the back side) are presented and discussed.

\section{EXPERIMENTAL APPARATUS}

The experiments were carried out in a perspex cylindrical tube of $5.5 \times 10^{-2} \mathrm{~m}$ inner diameter. A sketch is given in Fig. 1. The experiments were done as follows. First the tube is almost completely filled with a solution of glycerine in water, leaving a small region A occupied with air. Subsequently the tube is sealed at the upper end with a diaphragm and the region A with help of a vacuum pump brought to a pressure below atmospheric pressure. Then bubbles are supplied from below by means of a large number of capillary tubes. When the mixture is stationary, the mean volumetric gas content is determined by measuring the static pressure difference between two points along the tube located at a distance of $1.4 \mathrm{~m}$. A photograph of the mixture is taken from which the bubble size can be determined. In Fig. 2 such a photograph is shown. 


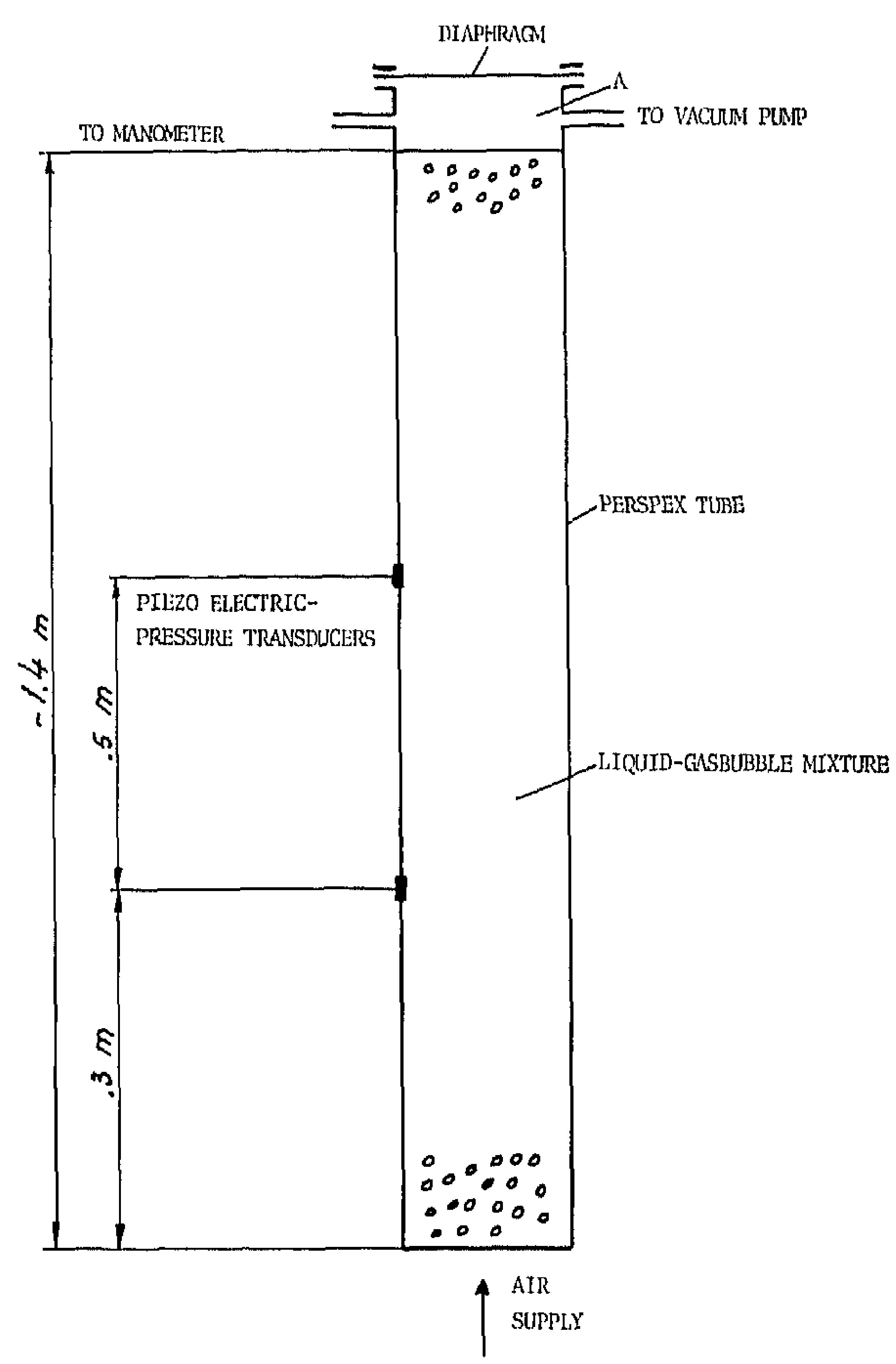

FIG. 1. Setup for experiments on shock waves in a bubble-liquid mixture.

The device by which the bubbles are supplied guarantees that in one experiment the bubbles have almost all the same size prior to the shock passage. The addition of glycerine is to keep the relative velocity due to buoyancy (the tube is in vertical position) as small as possible. The undisturbed volumetric gas content and bubble size thus determined, a shock wave is produced by puncturing the seal covering the tube and thereby admitting air of atmospheric pressure. After travelling some distance along the tube the shock becomes practically stationary and passes pressure transducers on two locations, a distance of $0.5 \mathrm{~m}$ apart. These transducers record the pressure changes in the mixture brought about by the passage of the shock. From the time-interval between the passage of the first and the second transducer the speed of the shock wave can be deduced. For some experiments a photograph of the shock wave was taken, after the propagation speed was determined. For this purpose a camera was focused on a certain part of the tube. The time of arrival of the shock at this part after passage of the first pressure transducer can be estimated sufficiently accurately from the measured speed of propagation. With this knowledge it was possible to trigger the camera with a signal produced by the first pressure transducer, allowing for a time delay. 


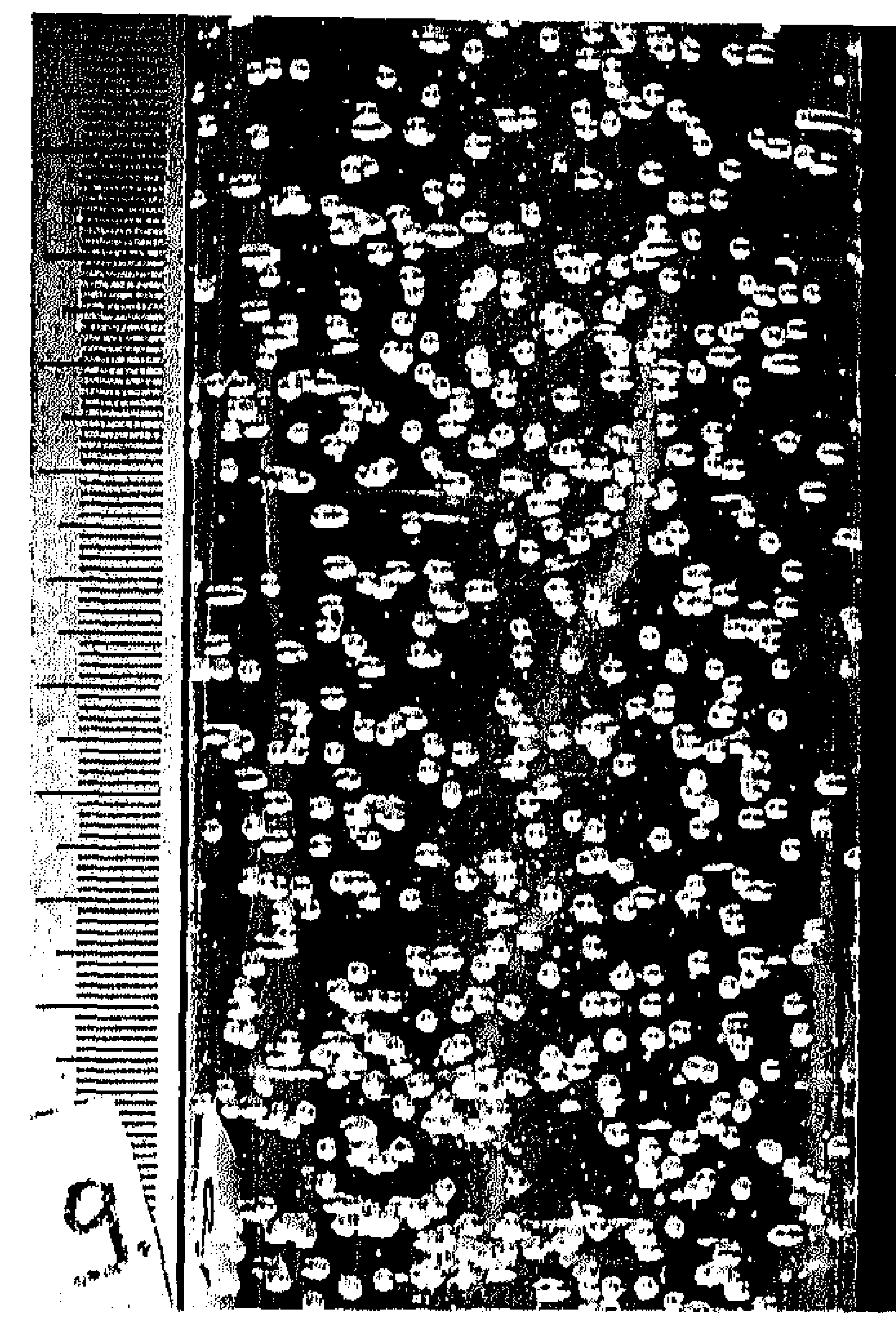

FIG. 2. Photograph of bubble-liquid mixture before the passage of a shock wave. From this type of photograph the bubble size is determined. $R_{0}=1.05 \times 10^{-3} \mathrm{~m} ; \beta_{0}=0.875 \times 10^{-2}$.

\section{RESULTS AND DISCUSSION}

In this section we present results of the experiments described in the previous section and compare these with theory. For a detailed account of the theory we refer to [1]. Here we give those elements which are necessary for a discussion of the experimental results.

\section{SPEED OF PROPAGATION OF THE SHOCK WAVE}

We assume that far in front of and far behind the shock wave the bubbles move with the fluid. Further we assume that in these regions the pressure in the air bubbles is the same as in the fluid. Denoting pressure with $p$, fluid density with $\rho_{f}$, gas concentration with $\beta$, shock velocity with $U$, velocity in mixture with $u$, and indicating quantities in front of the shock with 0 , those behind the shock with 1 , we have

$$
\begin{aligned}
\rho_{f}\left(1-\beta_{0}\right) U & =\rho_{f}\left(1-\beta_{1}\right)\left(U+u_{1}\right), \\
p_{0}+\rho_{f}\left(1-\beta_{0}\right) U^{2} & =\rho_{f}\left(1-\beta_{1}\right)\left(U+u_{1}\right)^{2}+p_{1}, \\
\frac{p_{1}}{p_{0}} & =\left(\frac{\beta_{0}}{\beta_{1}}\right)^{\gamma} .
\end{aligned}
$$

The first and second of these relations express conservation of mass and momentum across the shock. The third relation is used instead of an energy equation. In [1] isothermal conditions are assumed as in the work of Campbell and Pitcher [2], Eddington [3] and others. In that case $\gamma=1$. In the opposite limit of adiabatic behaviour of the air in the bubbles $\gamma=1.4$. 
From the above relations it follows that, for $\beta_{0} \ll 1$, which condition is fulfilled in all of our experiments,

$$
\frac{\rho_{f} U^{2} \beta_{0}}{p_{0}}=\frac{\left(p_{1} / p_{0}\right)-1}{1-\left(p_{0} / p_{1}\right)^{1 / \gamma}}
$$

It is of interest to note that $\left(p_{0} / \rho_{f} \beta_{0}\right)^{1 / 2}$ is (see [4]) the speed of sound $c$ in a bubbly mixture in the limit of infinite wavelength and under isothermal conditions. Introducing the associated Mach number $M_{\text {is }}$ by $U /(c)_{\text {is }}$ we have from (4) under isothermal conditions

$$
M_{\mathrm{is}}^{2}=\frac{p_{1}}{p_{0}} .
$$

The sound velocity corresponding with adiabatic behaviour is $\left(c^{2}\right)_{\text {ad }}=1.4 p_{0} / \rho_{f} \beta_{0}$. Therefore for adiabatic behaviour (5) may be written as

$$
M_{\mathrm{ad}}^{2}=\frac{1}{1.4} \frac{\left(p_{1} / p_{0}\right)-1}{1-\left(p_{0} / p_{1}\right)^{5 / 7}}
$$

In the experiments the time $t_{1}$ of travelling of the shock wave was measured between two points at distance $z_{1}=60 \mathrm{~cm}$ and $z_{2}=110 \mathrm{~cm}$ from the upper free surface of the mixture. Because the undisturbed values of $p_{0}$ and $\beta_{0}$ are affected by gravity, $U$ is not constant between these points. Therefore it was not possible to compare directly $U$ with its theoretical value. The experimentally obtained result is

$$
\left(t_{1}\right)_{\mathrm{exp}}=\int_{z_{1}}^{z_{2}} \frac{d z}{U(z)}
$$

For a comparison we have to calculate $t_{1}$ also from the theory. In a given experiment we measured the pressure increase $\Delta p$ across the shock, which did not change value between $z_{1}$ and $z_{2}$, and the pressure $p_{0}^{*}$ in the air occupied space A in Fig. 1. The distribution of $\beta_{0}(z)$ is isothermal so that when $\bar{\beta}_{0}$ is the measured mean value of $\beta_{0}$ along the tube, we have

$$
\beta_{0}(z)=\frac{\rho_{f} g L \bar{\beta}_{0}}{\ln \left(1+\frac{\rho_{f} g L}{p_{0}^{*}}\right)}\left(p_{0}^{*}+\rho_{f} g z\right)=\frac{B \bar{\beta}_{0}}{p_{0}^{*}+\rho_{f} g z}
$$

where $L$ is the length of the tube.

Then from (4) it follows that, with $p_{0}=p_{0}^{*}+\rho_{f} g z$ and $p_{1}=p_{0}^{*}+\rho_{f} g z+\Delta p$,

$$
U^{2}(z)=\frac{\Delta p\left(p_{0}^{*}+\rho_{f} g z\right)}{\rho_{f} B \tilde{\beta}_{0}\left\{1-\left(\frac{\left(p_{0}^{*}+\rho_{f} g z\right)}{p_{0}^{*}+\rho_{f} g z+\Delta p}\right)^{1 / \nu}\right.} .
$$

Hence, the theoretical travelling time of the shock is

$$
\left(t_{1}\right)_{\text {theory }}=\left(\frac{\rho_{f} B \bar{\beta}_{0}}{\Delta p}\right)^{1 / 2} \int_{z_{1}}^{z_{2}}\left[\frac{1-\left\{\frac{p_{0}^{*}+\rho_{f} g z}{p_{0}^{*}+\rho_{f} g z+\Delta p}\right\}^{1 / \nu}}{p_{0}^{*}+\rho_{f} g z}\right]^{1 / 2} d z
$$


Table 1. Values of Pertinent Quantities in Shock Propagation, as obtained IN THE EXPERIMENTS

\begin{tabular}{|c|c|c|c|c|c|}
\hline Experiment & $\frac{p_{1}}{p_{0}}$ & $\begin{array}{c}10^{2} d \\
\mathrm{~m}\end{array}$ & $\begin{array}{l}10^{3} R_{0} \\
\mathrm{~m}\end{array}$ & $10^{2} \beta_{0}$ & $\frac{d\left(\beta_{0}\right)^{ \pm}}{R_{0}}\left(1-\frac{1}{M_{\mathrm{ad}^{2}}}\right)^{\frac{1}{2}}$ \\
\hline 1 & 1.21 & 10.4 & 1.06 & 1.38 & 4.46 \\
\hline 2 & 1.21 & 11.4 & 0.9 & 0.74 & 4.21 \\
\hline 3 & 1.30 & 7.8 & 1.1 & 1.68 & 4.14 \\
\hline 4 & 1.30 & 7.5 & 0.98 & 1.1 & 3.60 \\
\hline 5 & 1.30 & 6.3 & 0.9 & 0.74 & 2.67 \\
\hline 6 & 1.40 & 5 & 1.25 & 2.04 & 2.88 \\
\hline 7 & 1.40 & 5.2 & 0.98 & 1.4 & 3.16 \\
\hline 8 & 1.40 & 5.1 & 0.95 & 0.96 & 2.66 \\
\hline 9 & 1.56 & 4.5 & 1.23 & 2.33 & 3.19 \\
\hline 10 & 1.56 & 4.2 & 1 & 1.68 & 3.10 \\
\hline 11 & 1.56 & 4.5 & 1 & 1.33 & 2.93 \\
\hline 12 & 1.70 & 3.8 & 1.35 & 2.75 & 2.82 \\
\hline 13 & 1.70 & 4.1 & 1.08 & 1.97 & 3.24 \\
\hline 14 & 1.70 & 3.7 & 1 & 1.61 & 2.86 \\
\hline 15 & 1.93 & 3 & 1.35 & 3.1 & 2.63 \\
\hline 16 & 1.93 & 3.2 & 1.09 & 2.25 & 2.92 \\
\hline 17 & 1.93 & 3.2 & $1^{1}$ & 1.82 & 2.88 \\
\hline 18 & 2.23 & 2.6 & 1.4 & 3.55 & 2.44 \\
\hline 19 & 2.23 & 3.7 & 1.12 & 2.62 & 3.76 \\
\hline 20 & 2.23 & 2.9 & 1.04 & 2.19 & 2.93 \\
\hline 21 & 2.54 & 2.1 & 1.45 & 4.16 & 2.24 \\
\hline 22 & 2.54 & 2.6 & 1.13 & 3.11 & 2.99 \\
\hline 23 & 2.54 & 3.3 & 1 & 1.47 & 2.99 \\
\hline 24 & 3.06 & 2 & 1.50 & 4.69 & 2.33 \\
\hline 25 & 3,06 & 2.6 & 1.05 & 3.05 & 3.42 \\
\hline 26 & 3.06 & 3 & 1 & 1.91 & 3.24 \\
\hline 27 & 3.79 & 2.1 & 1.56 & 6.04 & 2.73 \\
\hline 28 & 3.79 & 2.4 & 1.1 & 3.45 & 3.35 \\
\hline 29 & 3.79 & 2.1 & 1 & 2.48 & 2.81 \\
\hline 30 & 4.95 & 2.4 & 1.54 & 5.81 & 3.28 \\
\hline 31 & 4.95 & 2.1 & 1.30 & 4.8 & 3.12 \\
\hline 32 & 4.95 & 2.2 & 0.9 & 3.43 & 3.88 \\
\hline
\end{tabular}

In Table 1 are given, for thirty-two experiments, the values of $p_{1} / p_{0}$ and $\beta_{0}$, pertaining to the passage of shock waves at the first pressure transducer, located at $z=z_{1}$. The values in the table for $p_{1} / p_{0}$ and $\beta_{0}$ are corrected for gravity effects. In Table 2 are given for each of these experiments the value of the measured time $\left(t_{1}\right)_{\exp }$ of travelling of the shock wave between $z=z_{1}$ and $z=z_{2}$ and also $\left(t_{1}\right)_{\text {theory }}$ calculated numerically from (9) for adiabatic behaviour, $\gamma=1.4$.

Taking into account the experimental errors, especially the one occurring in the measurement of the pressure rise $\Delta p$ across the shock wave, we may conclude from comparison of $\left(t_{1}\right)_{\mathrm{exp}}$ with $\left(t_{1}\right)_{\text {theory }}$ that the shocks travel at a speed predicted by the adiabatic Hugoniot relations (1)-(3). Calculations carried out with (8) show that over the distance $z_{2}-z_{1}=$ $0.5 \mathrm{~m} U$ varies less than $8 \%$. Therefore it makes sense to compare $U\left(z_{1}\right)$ as calculated from (8) with an averaged speed $\bar{U}$ obtained from the measured $t_{1}$ by writing

$$
\left(t_{1}\right)_{\mathbf{e x p}}=\frac{z_{2}-z_{1}}{\bar{U}}
$$

H.M.T. $-\mathrm{X}$ 
Table 2. Experimental and Theoretical Values for the Velocity of PropagaTION OF SHOCK WAVES

\begin{tabular}{|c|c|c|c|c|}
\hline Experiment & $\begin{array}{l}10^{3}\left(t_{1}\right)_{\exp } \\
\quad \text { sec }\end{array}$ & $\begin{array}{c}10^{3}\left(t_{1}\right)_{\text {theory }} \\
\text { sec }\end{array}$ & $\begin{array}{c}(O)_{\exp } \\
\mathrm{m} / \mathrm{s}\end{array}$ & $\begin{array}{c}(U)_{\text {theory }}, \\
\mathrm{m} / \mathrm{s}\end{array}$ \\
\hline $\begin{array}{r}1 \\
2 \\
3 \\
4 \\
5 \\
6 \\
7 \\
8 \\
9 \\
10 \\
11 \\
12 \\
13 \\
14 \\
15 \\
16 \\
17 \\
18 \\
19 \\
20 \\
21 \\
22 \\
23 \\
24 \\
25 \\
26 \\
27 \\
28 \\
29 \\
30 \\
31 \\
32\end{array}$ & $\begin{array}{l}4.8 \\
3.5 \\
5.1 \\
4 \\
3.2 \\
6 \\
4.8 \\
3.9 \\
6.6 \\
5.9 \\
5 \\
7.3 \\
6.1 \\
5.4 \\
8.2 \\
7 \\
6.2 \\
8.8 \\
7.5 \\
6.9 \\
9.4 \\
7.8 \\
5.3 \\
9.8 \\
7.7 \\
5.9 \\
10.8 \\
8.4 \\
7 \\
10.4 \\
9.4 \\
8.1\end{array}$ & $\begin{array}{c}4.9 \\
3.6 \\
5.49 \\
4.47 \\
3.66 \\
6.09 \\
5.05 \\
4.19 \\
6.48 \\
5.52 \\
4.91 \\
7.11 \\
6.04 \\
5.47 \\
7.52 \\
6.43 \\
5.8 \\
8.01 \\
6.91 \\
6.33 \\
8.73 \\
7.58 \\
5.26 \\
9.22 \\
7.49 \\
5.96 \\
10.38 \\
7.95 \\
6.77 \\
10.15 \\
9.28 \\
7.9\end{array}$ & $\begin{array}{c}104.2 \\
142.9 \\
98 \\
125 \\
156.2 \\
83.3 \\
104.2 \\
128.2 \\
75.8 \\
84.7 \\
100 \\
68.5 \\
82 \\
92.6 \\
61 \\
71.4 \\
80.6 \\
56.8 \\
66.7 \\
72.5 \\
53.2 \\
64.1 \\
94.3 \\
51 \\
64.9 \\
84.7 \\
46.3 \\
59.5 \\
71.4 \\
48.1 \\
53.2 \\
61.7\end{array}$ & $\begin{array}{c}97.9 \\
133.6 \\
88.4 \\
108.7 \\
132.6 \\
79.6 \\
96 \\
115.8 \\
74.7 \\
87.7 \\
98.5 \\
67.5 \\
79.9 \\
88.3 \\
64.1 \\
74.9 \\
83 \\
59.9 \\
69.5 \\
75.8 \\
54.7 \\
63 \\
90.8 \\
51.5 \\
63.4 \\
79.6 \\
45.4 \\
59.2 \\
69.6 \\
45.8 \\
50.8 \\
58.8\end{array}$ \\
\hline
\end{tabular}

These values are given in Table 2 and show good agreement. Finally in Fig. 3 the relation (4) giving $\rho_{S} U^{2} \beta_{0} / p_{0}$ as a function of $p_{1} / p_{0}$ is drawn for the isothermal case, $\gamma=1$, and the adiabatic case, $\gamma=1.4$. For comparison, the corresponding values found in the experiments are presented in Fig. 3. Here for $U$ the value following from (10) is used. Here too we observe good agreement with the theory for adiabatic behaviour. It must be noticed that the discrepancy for very weak shocks is due to the fact that these were perhaps not stationary when passing the first pressure transducer. From this comparison of theory and experiment, in particular from $\left(t_{1}\right)_{\text {exp }}$ with $\left(t_{1}\right)_{\text {theory }}$ we conclude that the gas content of the mixture behaved adiabatically during the passage of a shock wave. At first sight this seemed rather surprising because Campbell and Pitcher [2] and Eddington [3] found excellent agreement with the isothermal relation (5). However, upon looking closer, our result can be explained. The assumption of isothermal behaviour in [1] was based on Plessets [5] result that oscillations of a bubble are isothermal both for low and high frequencies and adiabatic only in an intermediate range. For the bubbles in our experiments, having a radius of order of $1 \mathrm{~mm}$, typical frequencies are, however, just in this intermediate range, as may be shown as follows. 


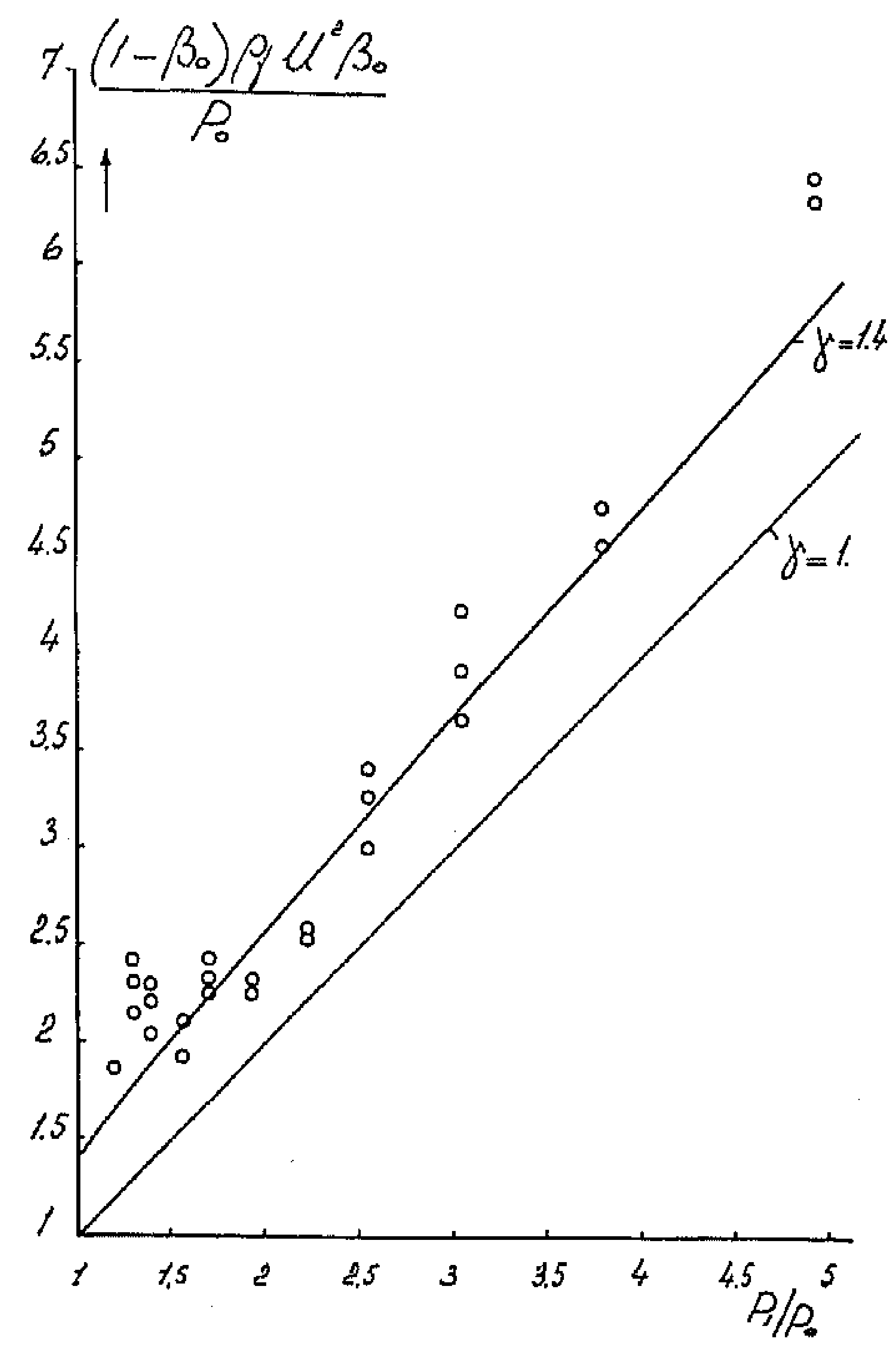

FIG. 3. Continuous lines, the theoretical relation (4) for $\gamma=1$ and $\gamma=1.4$. Circles, measurements, using $\left(t_{1}\right)_{\exp }=\left(z_{2}-z_{1}\right) / \bar{U}$.

For a shock velocity of order $10^{2} \mathrm{~m} / \mathrm{s}$ and shock-thickness of order $10^{-2} \mathrm{~m}$ (typical for our experiments) a typical frequency is $10^{4}$. The associated acoustic wavelength in air if of order $10^{-2} \mathrm{~m}$, which is an order of magnitude larger than the bubble size. Hence acoustical variations of pressure and temperature within a bubble may be neglected. The thermal penetration depth in the bubble is of order $(D / \omega)^{1 / 2}$ for angular frequency $\omega$ and thermal diffusion coefficient $D, 18 \times 10^{-6} \mathrm{~m}^{2} / \mathrm{s}$ for air. With a frequency of $10^{4}$, this amounts to $10^{-5} \mathrm{~m}$ which is negligibly small with respect to the bubble size, which is of order $10^{-3} \mathrm{~m}$. The behaviour of the air bubbles during the passage of the shock will therefore be adiabatic. In the mentioned experiments by Campbell and Pitcher isothermal behaviour was found. One reason for this is that they used considerably smaller bubbles, of order $10^{-4} \mathrm{~m}$. Another reason is that they reported on shock waves with (mostly) higher pressure ratios. It follows from the equation (4) that for large values of $p_{1} / p_{0}$ the "isothermal" relation (5) holds approximately for any value of $\gamma$.

\section{THICKNESS OF SHOCK WAVE}

Within the shock the local mean pressure $p$ in the mixture differs from the pressure $p_{g}$ in the bubble. These are related by

$$
p_{a}-p=\rho_{f}\left\{R \frac{d^{2} R}{d t^{2}}+\frac{3}{2}\left(\frac{d R}{d t}\right)^{2}+\frac{4 v}{d} \frac{d R}{d t}\right\}
$$

where $R$ denotes bubble radius and $\nu$ is the kinematic viscosity of the liquid. The difference $p_{g}-p$ is partly due to radial acceleration of fluid with respect to a bubble [the first two 
terms in the right-hand side of (11)], partly to viscous forces associated with the radial motion [last term in the right-hand side of (11)]. The local bubble radius $R$ is connected with the volumetric gas content $\beta$ by

$$
\beta=\frac{4}{3} \pi n R^{3} \text {. }
$$

$n$ is the number density of the bubbles and can, for $\beta_{0} \ll 1$, be considered as constant. Within the shock we have

$$
\rho_{f}(1-\beta)(U+u)=\rho_{f}\left(1-\beta_{0}\right) U,
$$

expressing mass conservation, and the momentum equation

$$
p+\rho_{f}(1-\beta)(U+u)^{2}=p_{0}+\rho_{f}\left(1-\beta_{0}\right) U^{2} .
$$

In [1] also the Kelvin impulse of the fluid associated with relative translational motion of the bubbles with respect to the fluid is taken into account, as well as the viscous friction due to this relative motion. It was shown in [1] that these effects are of minor importance when $\beta$ is small except for very weak shocks, or, more precisely, for shocks with strength of order $\beta$. Combination of (11)-(14) gives for isothermal behaviour

$$
\left(\beta_{0}-\beta\right)\left(\beta_{1}-\beta\right)=\frac{\bar{R}^{2}}{3} \frac{d^{2} \beta}{d x^{2}}+\frac{4}{3} \frac{\nu}{U} \frac{d \beta}{d x} .
$$

The coordinate $x$ runs along the shock from the low-pressure side $(x \rightarrow-\infty)$ to the highpressure side $(x \rightarrow \infty)$. The right-hand side of (15) stems from linearization of the terms in the right-hand side of (11). $\vec{R}$ is the bubble radius with respect to which the linearization is carried out. At the front of the shock where $\beta$ is near $\beta_{0}$ the solution of (15) behaves like

$$
\beta_{0}-\beta \sim \exp \left[-\frac{2 \nu x}{U R_{0}^{2}}+x\left\{\left(\frac{2 \nu}{U R_{0}^{2}}\right)^{2}+3\left(\frac{\beta_{0}-\beta_{1}}{R_{0}^{2}}\right)\right\}^{1 / 2}\right]
$$

Because of all our experiments

$$
\underset{U R_{0}}{\nu} \ll\left(\beta_{0}-\beta_{1}\right)
$$

it follows that the front part of the shock is determined by convection and dispersion, and that at the front $\beta_{0}-\beta$ increases like

$$
\exp \left\{\frac{3\left(\beta_{0}-\beta_{1}\right)}{R_{0}^{2}}\right\}^{1 / 2} x
$$

From (13) and (14) it follows that $p-p_{0}=\rho_{f} U^{2}\left(\beta_{0}-\beta\right)$. Then, using (3) for $\gamma=1$ and (5) we obtain for the pressure in the front part of the shock

$$
p_{0}-p \sim \exp \left\{\frac{\left(M_{\mathrm{is}}{ }^{2}-1\right)^{1 / 2} \beta_{0}^{1 / 2} x}{R_{0} M_{\mathrm{is}}}\right\} .
$$

For adiabatic behaviour, $\gamma=1.4$, the corresponding expression is

$$
p_{0}-p \sim \exp \left\{\frac{\left(M_{\mathrm{ad}}^{2}-1\right)^{1 / 2} \beta_{0}^{1 / 2} x}{R_{0} M_{\mathrm{ad}}}\right\}
$$




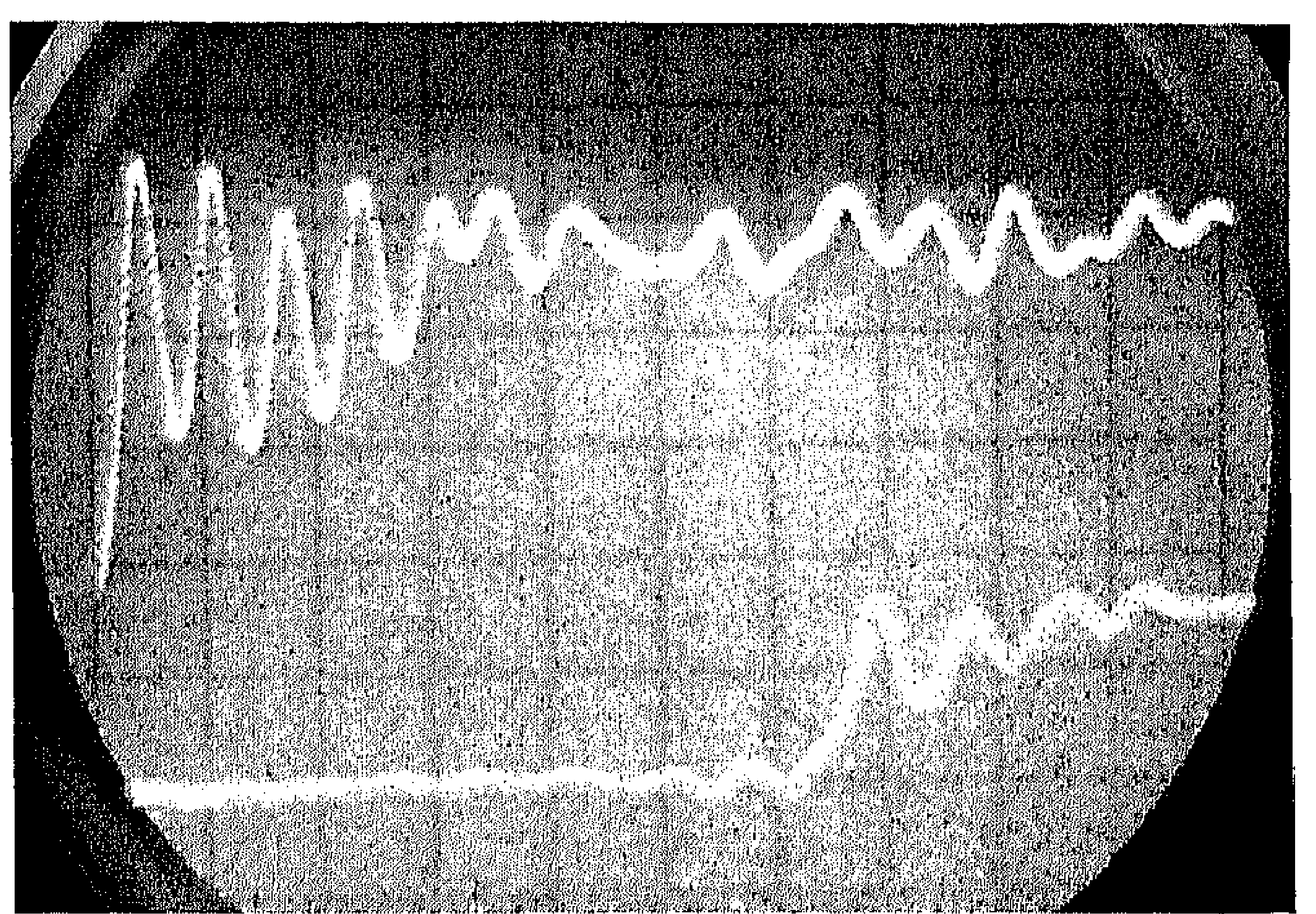

FIG. 4. Record of the pressure by the first pressure transducer during the passage of a shock wave for which $p_{1} / p_{0}=1.43 ; \beta_{0}=1.95 \times 10^{-2} ; R_{0}=1.15 \times 10^{-3} \mathrm{~m}$.

Because measurement of $U$ indicated adiabatic behaviour we compare the experimental values with (19), which predicts for the shock thickness $d$

$$
d \sim \frac{R_{0}}{\beta_{0}^{1 / 2}\left(1-\frac{1}{M_{\mathrm{add}}{ }^{2}}\right)^{1 / 2}}
$$

or

$$
d \frac{\beta_{0}^{1 / 2}}{R_{0}}\left(1-\frac{1}{M_{\mathrm{ad}}^{2}}\right)^{1 / 2}=\text { constant. }
$$

In Figs. 4 and 5 typical recordings are shown of the pressure behaviour in the bubble-liquid mixture during the passage of a shock wave. We took for the shock thickness $d$ the distance on these pressure records between the point where the pressure starts to rise till the top of the first crest.

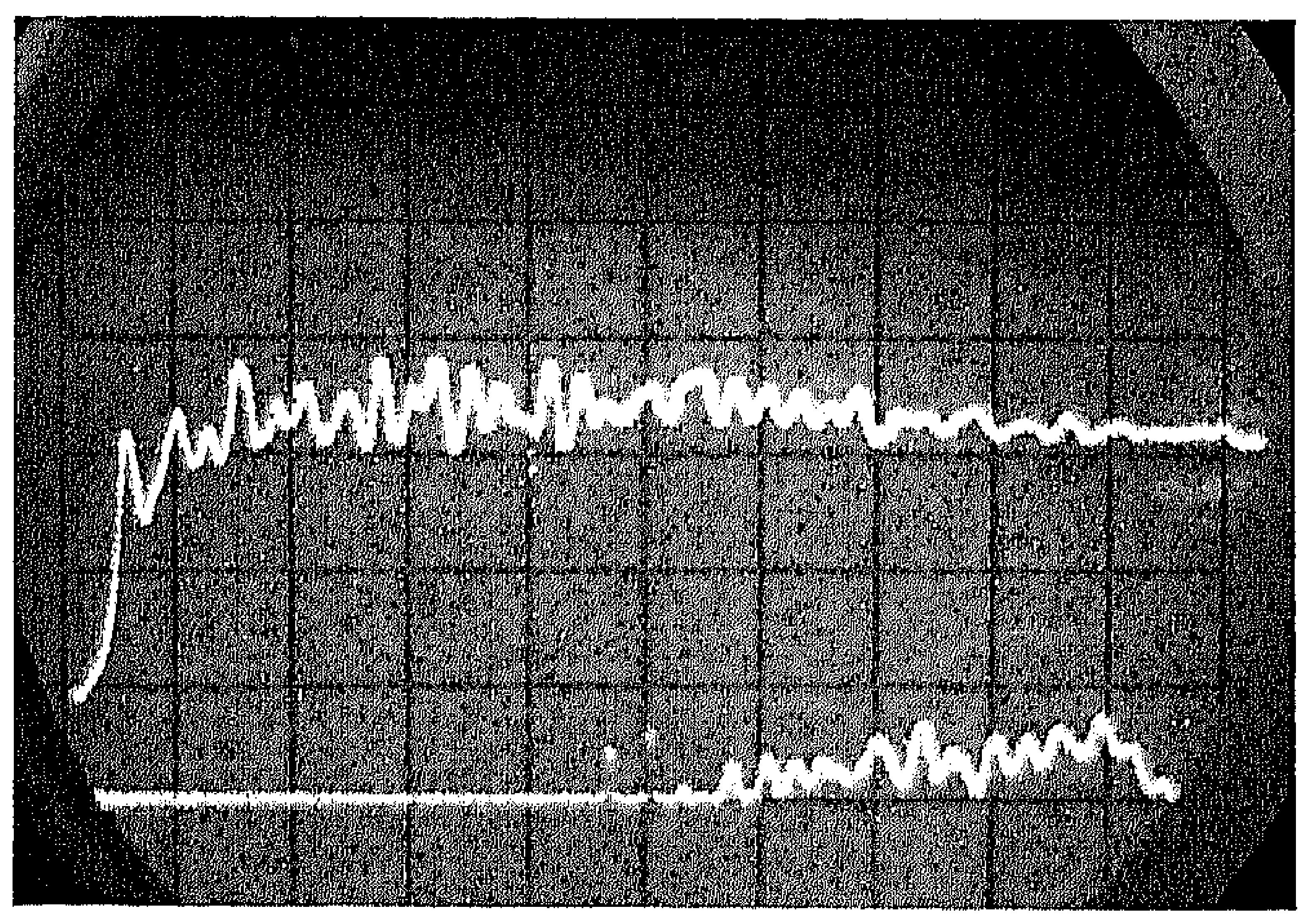

FIG, 5. Record of the pressure by the first pressure transducer during the passage of a shock wave for which $p_{1} / p_{0}=2.04 ; \beta_{0}=3.05 \times 10^{-2} ; R_{0}=1.38 \times 10^{-3} \mathrm{~m}$. 




FIG. 6. Verification of (21). Continuous line, $d \beta_{0}{ }^{1 / 2} \times 10^{3}=3.1\left(1-1 / M_{a d^{2}}\right)^{-1 / 2}$. Circles, experiment.

From the pressure records this thickness is given in terms of a time, $\Delta t$ say. This is converted in a distance $d$ by $d=U \Delta t$, where for $U$ we took the, experimentally determined, shock speed $(U)_{\text {exp }}$ in Table 2. The resulting shock thickness $d$ is given in the third column of Table 1 for each experiment. The theory predicts that the combination in the left-hand side of eqn. (21) is a constant. $d, \beta_{0}$ and $R_{0}$ are measured as described in the foregoing. Because satisfactory agreement was obtained with experiment in what concerns the relation (6), with $\gamma=1.4$, we inserted in (21) for $M_{n d}{ }^{2}$ the value following from (6) and the measured value of $p_{1} / p_{0}$. The relation (21) was verified in several ways. First in Table 1 , column 5 , the value of $d \beta_{0}{ }^{1 / 2} / R_{0}\left(1-1 / M_{\mathrm{ad}}{ }^{2}\right)^{1 / 2}$, as following from the experiments, is given. The value of this quantity, averaged over all experiments, is 3.05. Averaging over all but the first three experiments (bearing in mind that there the shock was possibly instationary) results in 3.13. In this set of twenty-nine experiments $d \beta_{0}{ }^{1 / 2} / R_{0}\left(1-1 / M_{\mathrm{ad}^{2}}\right)^{1 / 2}$ differs in twenty-four cases, $15 \%$ or less from 3.13 . This confirms the theory quite satisfactory because the experimental error, especially the error involved in measuring the bubble size from the photographs, is certainly not smaller than $15 \%$. As a further test we selected the six experiments for which $R_{0}=10^{-3}$ and draw in Fig. 6 the line $10^{3} d \beta_{0}{ }^{1 / 2}=3.1\left(1-1 / M_{\mathrm{ad}}{ }^{2}\right)^{-1 / 2}$. In the same figure we plotted also the experimentally obtained values of $10^{3} d \beta_{0}{ }^{1 / 2}$ against those of 3.1 $\left(1-1 / M_{\mathrm{nd}}{ }^{2}\right)^{-1 / 2}$, where the latter quantity is obtained from $p_{1} / p_{0}$ by using (6).

From Fig. 6 it follows that the experimental points fit the semi-theoretical curve (semitheoretical because the experimentally obtained constant 3.1 is used) very well. 


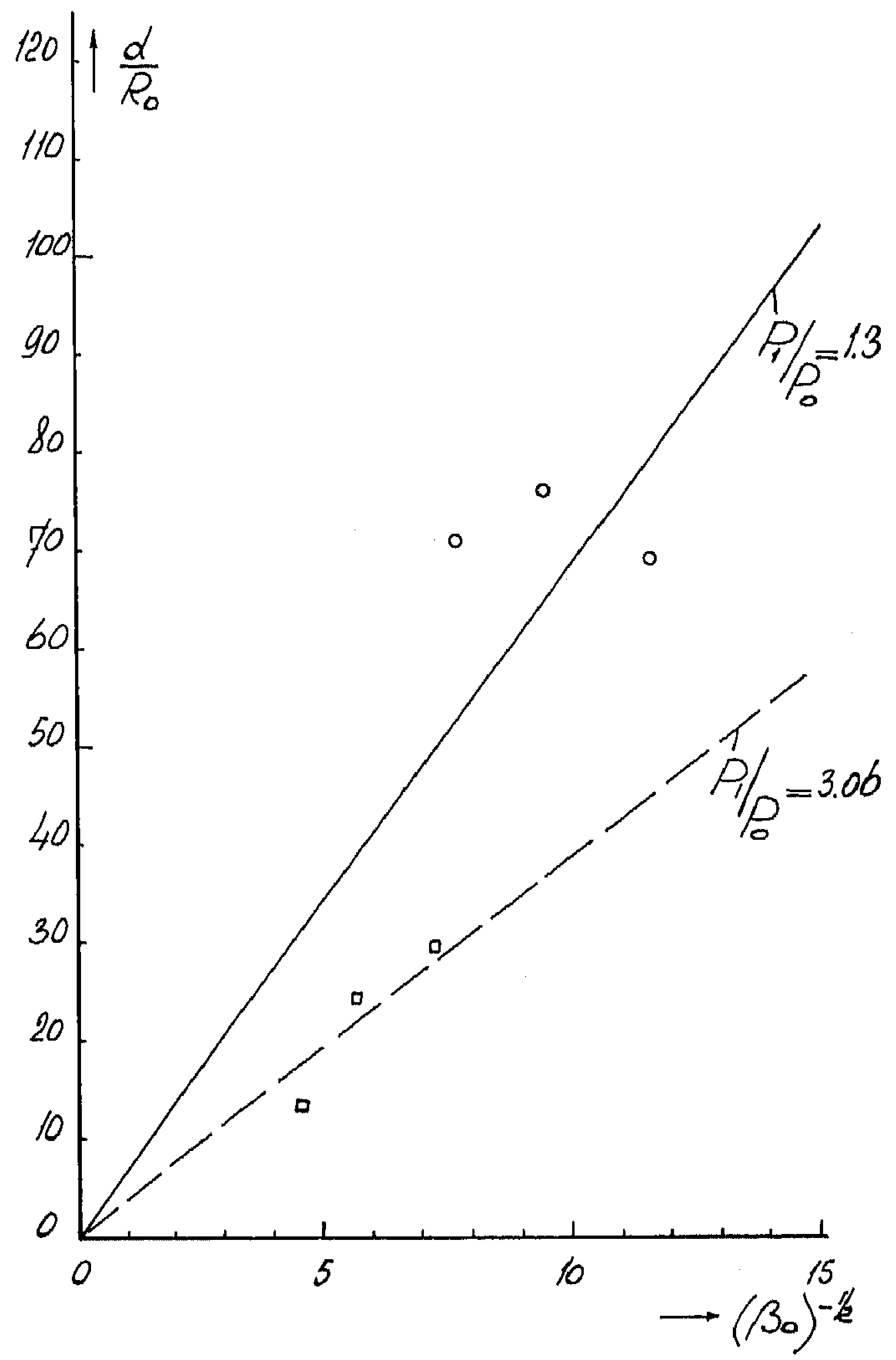

FIG. 7.

Finally, taking again the constant in (21) equal to 3.1, the lines for $d / R_{0}$ as function of $\beta_{0}{ }^{-1 / 2}$ are drawn in Fig. 7 for $p_{1} / p_{0}=1.3$ and $p_{1} / p_{0}=3.06$ (a constant value of $p_{1} / p_{0}$ implies a constant value of $M_{\mathrm{nd}}$ ). Also here the experimentally obtained values are given. This figure is less conclusive, because we had for each constant value of $p_{1} / p_{0}$ only three experiments.

In conclusion we may say that the relation (21) is by the experiments confirmed within the experimental accuracy. In recent work Noordzij [6] made an estimate of the theoretical value, following from approximate solution of eqn. (15), for the constant in the right-hand side of (21). Under circumstances as described here the theoretical value is 3.9.

\section{WAVES AT THE BACK SIDE OF THE SHOCK}

At the back side of the shock where $\beta$ is near $\beta_{1}$, the left-hand side of (15) may be linearized to give: 


$$
\frac{R_{1}^{2}}{3} \frac{d^{2} \bar{y}}{d x^{2}}+\frac{4}{3} \bar{y} \frac{d \bar{y}}{d x}+\bar{y}\left(\beta_{0}-\beta_{1}\right)=0
$$

where $\bar{y}=\beta-\beta_{1}$.

The solution of this equation tending to zero for $x \rightarrow \infty$ is, taking into account that $\left(\beta_{0}-\beta_{1}\right)^{1 / 2} \gg \nu / U R_{1}$,

$$
\bar{y} \sim \exp \left(-\frac{2 \nu x}{U R_{1}^{2}}\right) \exp \left\{\frac{3\left(\beta_{0}-\beta_{1}\right)}{R_{1}^{2}}\right\}^{1 / 2} x .
$$

This represents waves with wavelength

$$
\lambda=\frac{2 \pi R_{1}}{\left\{3\left(\beta_{0}-\beta_{1}\right)\right\}^{t / 2}} .
$$

This wavelength is of the same order of magnitude as the thickness of the shock wave (compare with (17)). For adiabatic behaviour the equation corresponding with (22) is

$$
\frac{R_{1}^{2}}{3} \frac{d^{2} \bar{y}}{d x^{2}}+\frac{4}{U} \frac{\nu}{d x}+\bar{y}\left\{\gamma\left(\beta_{0}-\beta_{1}\right)-\beta_{0}\left(\frac{\beta_{1}}{\beta_{0}}-M_{a d}{ }^{-2}\right)\right\}=0 .
$$

The corresponding wavelength is

$$
\lambda=\frac{2 \pi R_{1}}{\left[\gamma\left(\beta_{0}-\beta_{1}\right)-\beta_{0}\left(\frac{\beta_{1}}{\beta_{0}}-M_{\mathrm{nd}}{ }^{-2}\right)\right]^{1 / 2}} .
$$

The attenuation factor is $\exp -2 v x / U R_{1}{ }^{2}$ in both cases. On the pressure records in Fig. 4 and Fig. 5 waves are clearly visible. The wavelength is of the order of magnitude of the shock thickness indeed. Hence in this respect the theory is confirmed by the experiments. No agreement, however, exists between theory and experiment in what concerns the attenuation of these waves. The theoretical attenuation factor is $\exp -\left(2.10^{-4} x / R_{1}\right)$ for $\nu=10^{-5} \mathrm{~m}^{2} / \mathrm{s}$, $R_{1}=10^{-3} \mathrm{~m}, U=10^{2} \mathrm{~m} / \mathrm{s}$. Because $\lambda \sim d$ and $d \sim 30 R_{1}$ this is of order $\exp -10^{-2} x / \lambda$. According to this the wave would not be appreciably attenuated in, say, twenty wavelengths. From the pressure records it follows that considerable attenuation occurs over distances of that order. Theoretical work on the attenuation of acoustic waves through bubble-liquid mixtures shows that the main contribution stems from thermal conduction. Experiments in mixtures, where all bubbles are of the same size, show good agreement with these theoretical results, see Silberman [7], although the observed attenuation is systematically somewhat larger than the theoretically predicted one. Noordzij [6] has shown that the observed attenuation of the waves behind the shock, discussed here, can be, to a large extent, explained by taking thermal conduction within the bubbles into consideration.

\section{NOMENCLATURE}

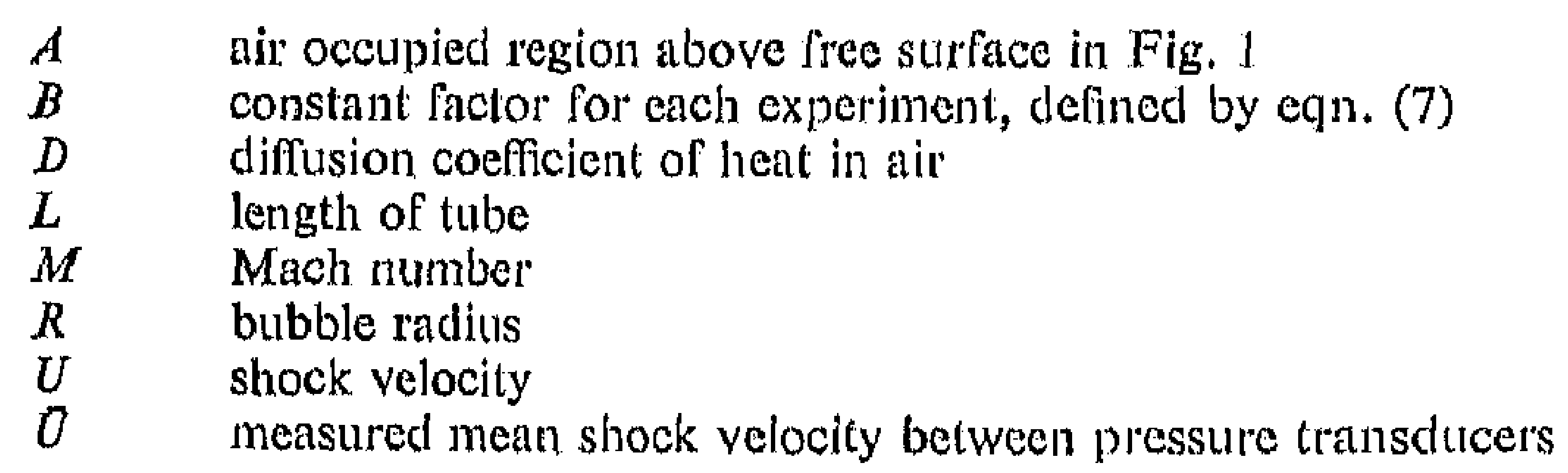




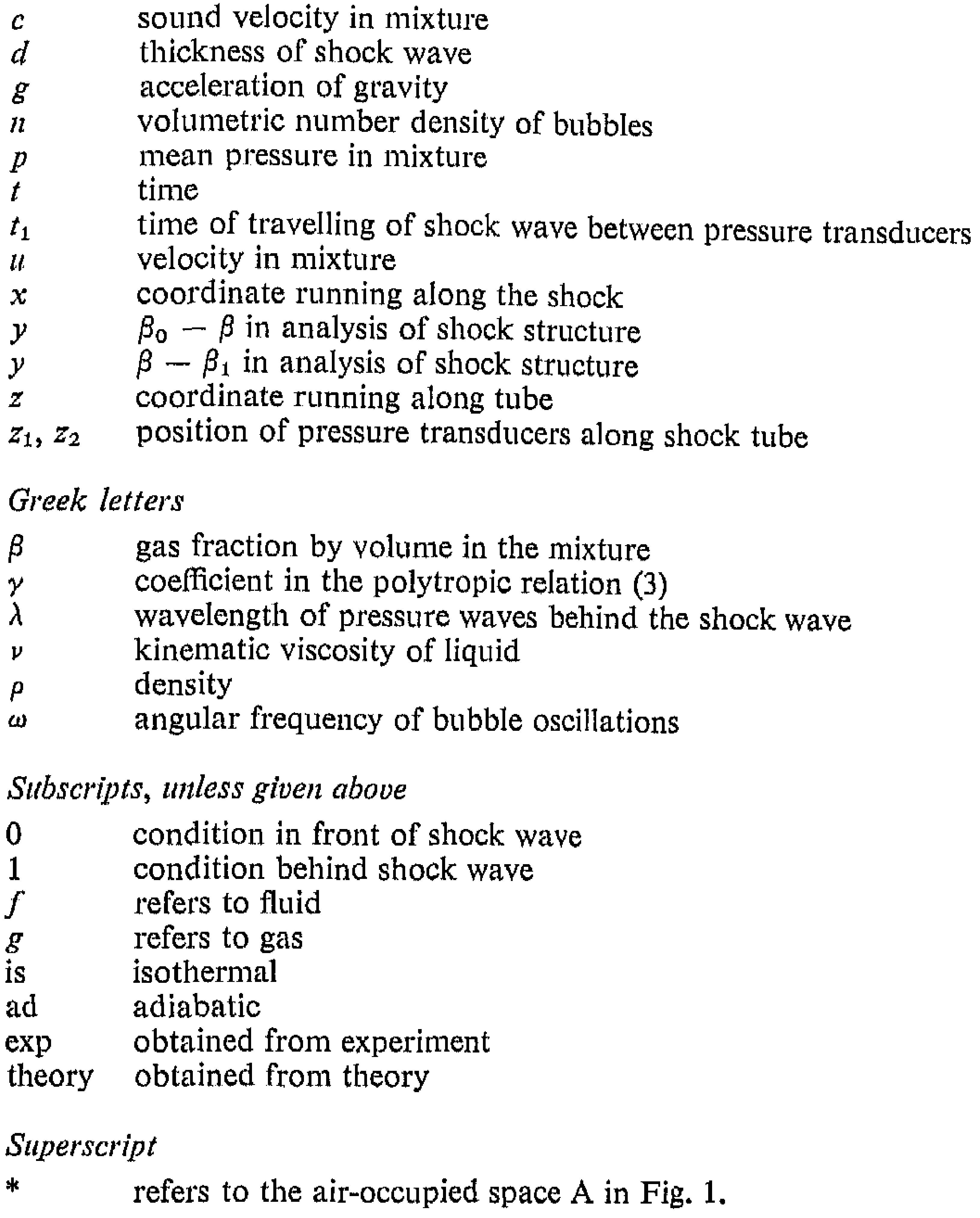

\section{REFERENCES}

1. L. van WiJnGaARDEN, On the structure of shock waves in liquid-bubble mixtures, Appl. Sci. Res. 22, 366 (1970).

2. I. J. Campbell and A. S. Pitcher, Shock waves in liquid containing gas bubbles, Proc. Royal Soc. A 243, 534 (1958).

3. R. EDDINGTON, Investigation of supersonic phenomena in a two-phase (liquid-gas) tunnel, AIAA J. 8, 1 (1970).

4. L. VAN WIJNGAARDEN, On the equation of motion for mixtures of liquid and gas bubbles, J. Fluid Mech. $33(3), 465(1968)$

5. M. S. Plesset, On bubble dynamics, in Cavitation in Real Liquids, ed. R. Davies, Elsevier, AmsterdamNew York, 1964.

6. L. NoordzIJ, Shock waves in bubble-liquid mixtures, Phys. Comm. Twente Inst. Techn. 1, 3 (1971). To be published also in Proc. IUTAM Symp. on Nonsteady Flow of Water at High Speeds, Leningrad (1971).

7. E. SilbERMAN, Sound velocity and attenuation in bubble mixtures measured in standing wave tubes, $J$. Acoust. Soc. Amer. 29, 8 (1957). 